\title{
Urinary Tract Infection in Children: Diagnostic and Prognostic Utility of Biomarkers
}

\author{
Samuel N. Uwaezuoke ${ }^{1, *}$ \\ ${ }^{1}$ MB, BS; FWACP, Honorary Consultant Pediatrician, Pediatric Nephrology Firm, Department of Pediatrics, University of Nigeria Teaching, Hospital (UNTH), Ituku-Ozalla, \\ Enugu \\ "Corresponding author: Samuel N. Uwaezuoke, Department of Pediatrics, UNTH Ituku-Ozalla, Postal code- 400001, Enugu. Tel: +234-8033248108, E-mail: \\ snuwaezuoke@yahoo.com
}

Received 2016 November 13; Revised 2017 March 31; Accepted 2017 May 04.

\begin{abstract}
In the diagnostic evaluation of urinary tract infection (UTI) in children, dipstick urinalysis and urine microscopy/culture are the initial steps. Although these investigations are not without their drawbacks, urine culture remains the gold standard. Biomarkers may also be used as diagnostic tools, predictors of complications, and renal parenchymal injury. This paper aims to review the studies reporting the utility of biomarkers in the diagnosis and prognostication of UTI. Using the key words- 'urinary tract infection', 'children', and 'biomarkers'- the PubMed database was searched for systematic reviews/meta-analyses and original studies, which were published within the past 25 years. Information was also gathered from relevant textbooks published within the same period. Serum procalcitonin, urine/serum interleukin 6 (IL-6) and 8 (IL-8), as well as urine neutrophil gelatinase-associated lipocalin (NGAL) are the major biomarkers of febrile UTI. In addition, procalcitonin and these cytokines are reported to be early predictors of vesicoureteric reflux (VUR) and renal parenchymal injury, while procalcitonin and IL-6 can also help to differentiate between lower UTI and upper UTI (pyelonephritis). Despite the wide range in the sensitivity and specificity of these biomarkers reported in the reviewed studies, the values are still acceptable. Some of these biomarkers may, in the future, obviate the need for invasive radiological tests, although their utility still require validation by further research. However, given the ability of imaging studies to detect structural abnormalities of the urinary tract, their use still remains relevant in the investigation of UTI despite the radiation risks associated with majority of them.
\end{abstract}

Keywords: Urinary Tract Infection, Children, Biomarkers

\section{Introduction}

Urinary tract infection (UTI) refers to any bacterial, viral, or fungal infection affecting any part of the urinary tract- either the upper tracts (pyelonephritis) or the lower tracts (cystitis/urethritis). In children, bacteria (especially coliform bacteria such as Escherichia coli) constitute the major etiologic agents; however, in immunocompromised children and premature babies, fungi are occasionally seen (1).

The clinical presentation of UTI varies with age as the symptoms are non-specific in the neonates and infants, while the classical symptoms of dysuria and frequency assume more prominence with increasing age, particularly in pre-schoolers (2). UTI ranks third on the list of common pediatric infections in developing countries (1) and is one of the most common causes of febrile illness in children $(3,4)$. Up to $11.3 \%$ of girls and $3.6 \%$ of boys will have had a UTI by the age of 16 years; recurrence of infection is a usual problem (5).

The health implications of UTI are enormous in children due to repeated involvement of the upper tracts that potentially lead to scar formation, which predisposes to hypertension and chronic kidney disease (CKD) (2). Thus, instituting early and appropriate treatment can prevent the development of these complications. In fact, the common mistake in the management of UTI in pediatric practice is the failure to establish the diagnosis promptly in the first place; there will either be a missed opportunity to prevent renal injury if UTI is not diagnosed at all, or unnecessary invasive tests if it is not correctly diagnosed (2).

In the diagnostic evaluation of UTI, dipstick urinalysis and urine microscopy/culture are the initial basic steps. Urine culture remains the 'gold standard' for confirming the infection. These investigations are not without their drawbacks. For instance, as a screening test, dipstick urinalysis (leucocyte esterase and nitrite tests) may give false negative or false positive results while a reliable urine culture result is highly dependent on the method and the aseptic technique of collecting the urine sample, which underscores the importance of a proper interpretation of a positive culture result.

Subsequently, novel biomarkers are emerging as alternative tools in the diagnosis of UTI (6-13), and as predictors of its complications such as vesico-ureteric reflux 
(VUR) (14-18) as well as renal parenchymal injury (19-22). Reports also show that febrile UTI in children activate an interleukin 6 (IL-6) and interleukin 8 (IL-8) responses, thus adding the estimation of these cytokines in the diagnostic trajectory of the disease $(23,24)$. Moreover, some of them have been found useful in differentiating between lower UTI and pyelonephritis (25). Therefore, the prospect of a rapid diagnostic tool with acceptable sensitivity and specificity makes the use of biomarkers an attractive option although the cheaper and more readily available urine culture still remains the gold standard. This paper aims to review the studies showing the utility of biomarkers in the diagnosis and prognostication of UTI.

\section{Literature Search Strategy}

Using the key words- 'urinary tract infection', 'children', and 'biomarkers'- PubMed database was searched for studies, which were published within the past 25 years. Systematic reviews/meta-analyses and original studies were included for appraisal in the present narrative review. Information was also gathered from relevant textbooks published within the same period.

\section{The Current Paradigm for Diagnostic Evaluation}

The bedrock of diagnostic evaluation in childhood UTI consists of microbial isolation and radiological evaluation of the urinary tract. While urine-screening tests essentially signal the presence of etiologic bacteria, urine culture is meant to isolate the organisms and test for their antibiotic sensitivity patterns. Furthermore, radiological investigations form part of the diagnostic armamentarium in children because urinary tract abnormalities often predispose to, or complicate episodes of UTI.

The conventional screening tests include the leukocyte esterase and nitrite tests. Although a positive leukocyte esterase test by dipstick urinalysis suggests the presence of pyuria, a urine microscopy is necessary to confirm the finding. Pyuria refers to the presence of $>10 \times 10^{6}$ white blood cells/L in an uncentrifuged urine specimen or $>5$ $\times 10^{6}$ white blood cells/L in a centrifuged urine specimen (2). Nevertheless, pyuria can also occur in other conditions other than a UTI such as urolithiasis and renal tuberculosis: the so-called 'sterile pyuria' (1). Its poor predictive value as an isolated result thus makes it unreliable for the presumptive diagnosis of UTI.

Positive nitrite test is based on the conversion of dietary nitrates to nitrite by coliform bacteria. Again, a negative test does not preclude a UTI, as it may indicate an infection with non-coliform bacteria such as Streptococcus fae- calis and Staphylococcus epidermidis. Secondly, inadequate dietary nitrate may result in a false negative test.

The Pediatrician is then confronted with critical decisions on key management options such as giving empirical antibiotics or performing these routine urine tests. This management dilemma led to the development of some global guidelines on UTI in children. One of them is the national institute of health and care excellence (NICE) guidelines, which was published in 2007, and have greatly influenced clinical practice with respect to the diagnosis, clinical management, and radiological investigation of the child with UTI (26). The other is the American academy of pediatrics (AAP) guidelines, which was first published in 1999, however, was revised in 2011 (27). In the NICE guidelines. The following management options have been recommended depending on the results of urine dipstick tests (Figure 1): if leukocyte esterase and nitrite tests are positive, antibiotic treatment for UTI is recommended, as well as urine culture if risk of serious illness is high or intermediate, and if there is past history of UTI; if leukocyte esterase test is negative and nitrite test is positive, antibiotic treatment and urine culture are recommended; if leukocyte esterase test is positive and nitrite test is negative, antibiotic treatment -only with good clinical evidence of UTI as well as urine culture and treatment (based on results) are recommended (26).

In the radiological evaluation of a child with UTI, the scope of investigations remains unresolved because of the invasive nature and radiation exposure of the tests as well as dearth of evidence to indicate that outcome is improved (2). There is now a paradigm shift from traditional protocols that employ age alone as the determinant for radiological investigations to protocols that enroll children when they are at high risk of renal injury (2). Nevertheless, the current NICE guidelines recommend that different radiological tests should be done according to the age of the child; whether there is good response to treatment within 48 hours; whether there is evidence of atypical UTI (seriously ill child, poor urine flow, abdominal or bladder mass, azotemia, septicemia, non-response to treatment within 48 hours and non-Escherichia coli UTI) and; whether there is evidence of recurrent UTI (26). The essential radiological tests are Ultrasonography (US) at the time of acute infection and within 6 weeks, Dimercapto-succinic acid (DMSA) scan at 4 - 6 months, and Mictuirating Cystourethrogram (MCUG). The initial US is supposed to detect severe structural abnormalities and urinary obstruction, bladder wall thickness and emptying, as well as renal defects; the DMSA scan is meant for the diagnosis of renal parenchymal injury; while MCUG is meant for identifying urethral abnormalities and VUR. For instance, in the NICE guidelines for radiological investigations following UTI in children aged 


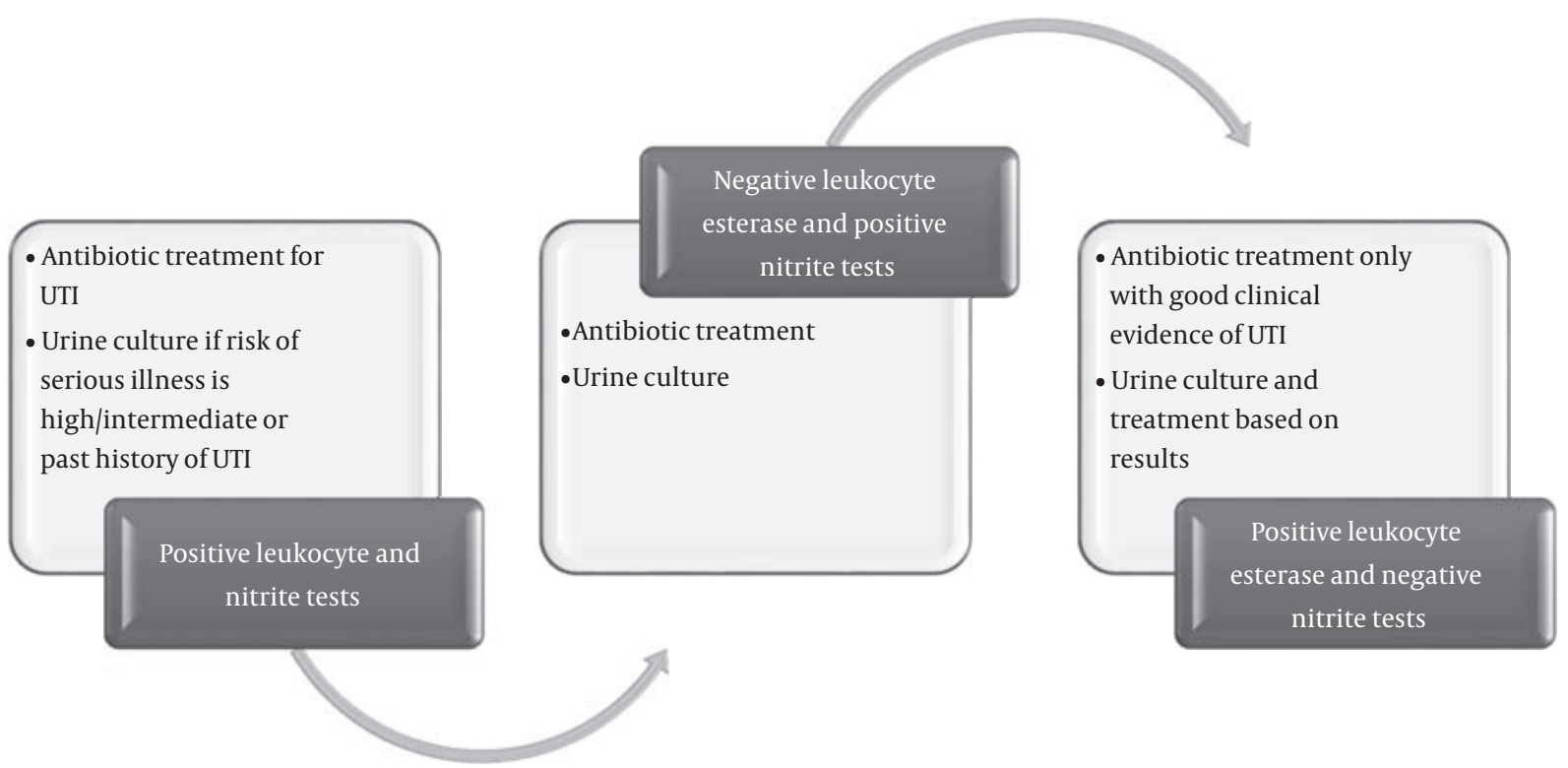

UTI, urinary tract infection; NICE, national institute for health and care excellence.

6 months to 3 years, initial and later US, DMSA scan at 4 6 weeks, and MCUG are not recommended if there is good response within 48 hours; for atypical UTI, initial US and DMSA scan at 4-6 weeks are recommended (the caveat being that later US can be done if there is non- Escherichia coli not responding well to treatment) while US within 6 weeks and MCUG are not recommended (the caveat being that MCUG can be done if dilatation is noted on US, and if there is poor urine flow, non-Escherichia coli UTI and family history of VUR); and for recurrent UTI, initial US and MCUG are not recommended while later US and DMSA scan at 4-6 weeks are recommended (26). The AAP guidelines recommend urinary tract imaging in a febrile infant or young children between the ages of 2 months and 2 years with a first documented UTI (27). The imaging studies typically comprise MCUG, as well as renal and bladder US (RBUS). In the revised AAP, routine MCUG is no longer recommended after the first episode of UTI because of radiation exposure, financial cost, and considerable test-related discomfort. However, MCUG is indicated if RBUS shows hydronephrosis, renal scar or VUR. Although both NICE and AAP guidelines have their strengths, which include high specificities for VUR, their obvious weaknesses are the high financial and radiation costs to the patient.

\section{Biomarkers as Diagnostic and Prognostic Tools in UTI}

Generally, proteomic biomarkers are potential tools for improving the diagnosis and prognostication in patients with kidney diseases as they ensure more accurate and earlier identification of renal pathology (28). The characteristic features of an ideal biomarker include its secretion by the injured cells and its organ specificity, its level being proportionate to the extent of injury, its early expression after a potentially reversible organ injury, its prompt reduction in level after injury to enable its use as a monitoring parameter for treatment, and its quick and reliable estimation (29). Furthermore, it is important to establish the sensitivity and specificity of each biomarker for UTI. The sensitivity of the biomarkers refers to their ability to detect patients who have a UTI (i.e. the proportion of the patients who test positive for UTI among those who have the disease) while their specificity refers to their ability to correctly detect patients without a UTI (i.e. the proportion of healthy children known not to have UTI who will test negative for it). Remarkably, procalcitonin and interleukins are the major biomarkers reported in the evaluation of the disease in children (Figure 2). 


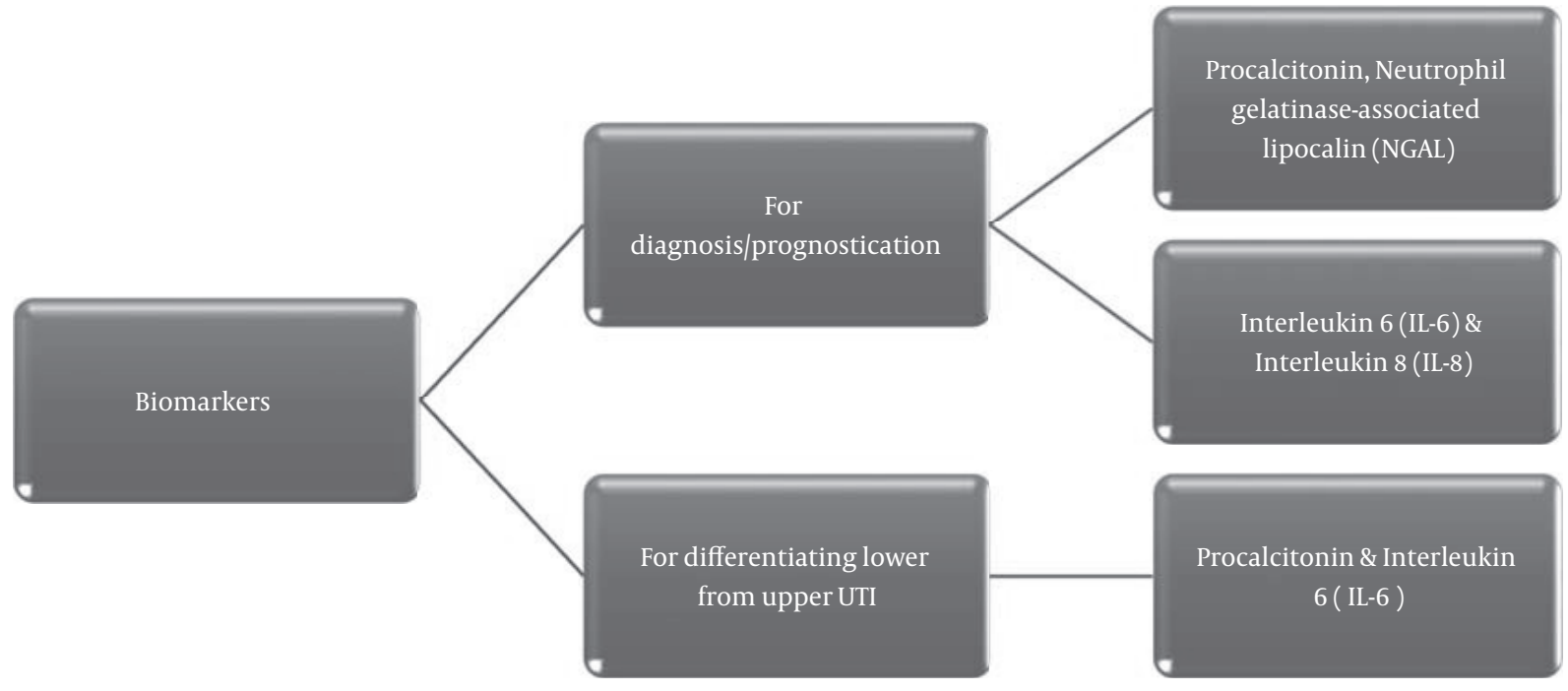

Figure 2. Summary of Current Biomarkers Used in the Diagnosis and Prognostication of Childhood UTI

\section{Interleukins (IL) and Acute Phase Reactants}

UTI stimulates local and systemic cytokine responses (30-34). In one study that compared the set of cytokines produced by uroepithelial cell lines and human peripheral blood monocytes in response to Escherichia coli, the authors demonstrated that bacteria activated a cytokine response in the epithelial cell lines and monocytes (30). Specifically, they found that the cell lines stained for IL-8 with a peak at 2 hours and IL- 6 with a peak at 6 hours after Escherichia coli stimulation. Peripheral blood monocytes also stained for the cytokines IL-1 $\alpha$, IL-1 $\beta$, IL-8, IL-6, and tumor necrosis factor- alpha (TNF- $\alpha$ ) after stimulation with the same organism. These findings were corroborated by another study, which compared the cytokine production of uroepithelial cell lines in response to gram-negative bacteria and inflammatory cytokines (31). Escherichia coli IL-1 $\alpha$ and TNF- $\alpha$ were found to activate the de novo synthesis and secretion of IL- 6 and IL-8 in uroepithelial cells: highlighting the role of these cells in cytokine-mediated responses during the early phases of infection (31). Furthermore, it has also been reported that Uropathogenic Escherichia coli elicits a host response that determines the severity of UTI, as specific adherence mechanisms permit the bacteria to initiate this process by targeting epithelial cells in the urinary tract mucosa (32). Other authors confirmed these observations in their report when they noted that the adherence of Uropathogenic Escherichia coli to mucosal sites in the urinary tract is followed by inflammation, including a mucosal cytokine response (33). The uroepithelial cells are activated to secrete IL-6, while IL-8, as the former, may cause the fever and acute phase response that accompany systemic UTIs, while the latter may function as a neutrophil chemoattractant (33). Finally, one report indicates that Escherichia coli elicited a mucosal IL-8 response in humans, and suggests the involvement of the cytokine in the onset of pyuria: concluding that uroepithelial cells may be an important source of IL-8 during UTI (34).

Other group of investigators focused on IL-6 response in 114 children with suspected UTI. The results of their study showed that infections of the urinary tract activate an IL-6 response in children, the magnitude of which is influenced by the properties of the infecting strain, such as the possession of P fimbriae (23). Given the fact that IL6 and IL-8 are expressed early after a UTI episode and the fact that they are secreted by uroepithelial cells, these cytokines have clearly fulfilled some of the 5 characteristics of an ideal biomarker.

The importance of acute phase reactants in the diagnosis of UTI has also been highlighted in the study done by Benson et al. (10). The authors showed that urine and serum IL-6 and IL-8 responses were higher in children with febrile UTI than in those with asymptomatic bacteriuria (10). By univariate analysis, the cytokine levels were found to be related to age, gender, VUR, renal scarring, urine leukocytes, acute phase reactants [such as C-reactive protein (CRP) and erythrocyte sedimentation rate (ESR)], as well as bacterial properties (such as P fimbriae). Multivariate analysis revealed that urine IL- 6 responses were higher in girls than boys, increased with age, and were positively correlated with CRP, ESR, serum IL-6, and urine leukocyte counts. The urine IL-8 response was not influenced by age, 
but was influenced by P fimbriae, and was related to ESR, CRP, urine leukocytes, as well as female gender. Thus, interleukins and acute phase reactants synergistically reflect the inflammation associated with UTI. The authors also concluded that cytokine responses to UTI vary with the severity of infection and that cytokine activation is affected by a variety of host and bacterial variables (10).

In a prospective, case-control study of children aged 0 to 12 years, Jantausch and co-workers also evaluated urinary IL-6 and IL-8 as biomarkers for UTI (35). The study control group consisted of febrile subjects with infections other than a UTI. At the time of admission, the authors found that there was a statistically significant difference between the median urinary IL- 6 concentration for subjects with proven bacterial UTI and that for controls. Similarly, the median urinary IL-8 concentration for the bacterial UTI group was significantly higher than that for the controls.

In their study, Sheu et al. compared the utility of serum and urine levels of IL- 6 and IL-8 for diagnosing acute pyelonephritis (24). They enrolled 78 children aged 1 to 121 months with a first episode of febrile UTI. Twelve healthy children, matched for age and sex, were used as controls. Serum and urine IL-6 and IL-8 were collected at presentation. Acute pyelonephritis was confirmed with DMSA scan. The initial value of serum and urine IL- 6 and IL-8 was significantly higher in patients with acute pyelonephritis than in those with lower UTI and in healthy controls. Remarkably, serum and urine IL- 6 had a higher sensitivity and specificity in the diagnosis of acute pyelonephritis compared to serum and urine IL-8 (24). Sensitivity and specificity for serum IL-6 was noted to be $88 \%$ and $83 \%$, respectively, while for urine IL-6, sensitivity and specificity was $86 \%$ and $81 \%$, respectively, using specific cut-off values in both instances.

In another study conducted in children aged less than 1 year, Gurgoze et al. showed that at the time of presentation, median serum IL-6 was higher in the pyelonephritis group compared with the lower UTI group (36). The diagnosis of pyelonephritis with serum IL-6 yielded a sensitivity of $88 \%$ and a specificity of $74 \%$. Interestingly, the researchers also reported that serum IL-1 $\beta$ had a sensitivity of $97 \%$ and a specificity of $59 \%$ for detecting pyelonephritis (36).

\section{Procalcitonin}

Procalcitonin had long been recognized as a biomarker of severe bacterial infection (37). In a prospective study of 80 children aged 1 month to 16 years of age, a group of investigators compared the procalcitonin level, CRP level and white blood cell count in children with pyelonephritis and lower UTI (38). There were statistically significant differences between the pyelonephritis and lower UTI groups for all 3 parameters; but only procalcitonin had a good correlation with increasing severity of changes noted on DMSA scan. Their findings are in keeping with those of Gürgöze et al. who noted that the median procalcitonin level obtained at presentation was significantly higher in the acute pyelonephritis group than in the lower UTI group (36).

In another prospective study of 64 children, Smolkin et al. demonstrated that in acute pyelonephritis, the median procalcitonin level was significantly higher than that obtained in lower UTI (39). Moreover, using a specific cutoff value, procalcitonin had a sensitivity of $94.1 \%$ and specificity of $89.7 \%$ for detecting pyelonephritis. Other workers also reported a similar diagnostic utility of procalcitonin as they showed that could serve as a marker of severity of acute pyelonephritis in children (40). Remarkably, a cut-off value of $0.8 \mathrm{ng} / \mathrm{mL}$ gave the best diagnostic accuracy with a sensitivity of $83.3 \%$ and specificity of $93.7 \%$ for the diagnosis of acute pyelonephritis. Nevertheless, other investigators were not able to demonstrate a significant correlation between renal parenchymal injury on one hand and procalcitonin level, CRP level, and white blood cell count on the other hand (41). In their study, the subjects were recruited based on presenting symptoms, while diagnosis of acute pyelonephritis was confirmed with DMSA scan.

In a retrospective hospital-based cohort study on children (aged 1 month to 4 years) who had a first febrile UTI, Leroy et al. reported that high procalcitonin was a strong and independent predictor of VUR, and could be used to identify low-risk patients in order to avoid unnecessary MCUG (14). The authors arrived at this conclusion based on their study findings, which showed that high procalcitonin was not only associated with VUR but that the relationship was stronger for high-grade reflux than for lowgrade reflux. In addition, high procalcitonin sensitivities were $85 \%$ and $92 \%$ for all-grade and high-grade reflux respectively, with only $44 \%$ specificity (14).

To corroborate these findings, Sun et al. also assessed the utility of procalcitonin as a biomarker for predicting high-grade VUR in young children with a first febrile UTI (16). Children aged less than 2 years and had a first febrile UTI, were prospectively evaluated at the time of admission using serum procalcitonin, renal US, DMSA renal scan, and MCUG. Similarly, the authors were able to establish the following: the significantly higher median procalcitonin level in children with VUR than in those without VUR, the sensitivity and negative predictive value of 94.3\% and 95.4\%, respectively for predicting high-grade VUR using procalcitonin, and $97.1 \%$ as well as $97.8 \%$, respectively using combined procalcitonin and US studies. When 
they further subjected their results to multivariate analysis and high procalcitonin values, abnormalities on US were found to be independent predictors of high-grade VUR. The authors then concluded that procalcitonin was a useful biomarker for the diagnosis of acute pyelonephritis and for the prediction of high-grade VUR in young children with first febrile UTI (16).

In Iran, Rahimzadeh et al. also investigated the role of serum procalcitonin as a predictor of VUR in children admitted for acute pyelonephritis (17). They noted that there was a significant relationship between high-grade VUR and higher levels of procalcitonin. For the diagnosis of high-grade VUR, the sensitivity of a procalcitonin level of $>0.31 \mathrm{ng} / \mathrm{mL}$ was $90 \%$ while the specificity was $32 \%$, making the researchers to conclude that serum procalcitonin level was a sensitive and promising predictor of high-grade VUR (17). In tandem with these findings were those of Mortazavi and Ghojazadeh who also evaluated the predictive value of procalcitonin in the diagnosis of VUR in 108 children with febrile UTI (18). In these children aged 2 months to 12 years, serum procalcitonin was estimated before commencing antibiotics, while standard MCUG was performed to detect VUR. When the authors compared procalcitonin levels with MCUG results, a sensitivity of $97 \%$ and a specificity of $75 \%$ were obtained at a procalcitonin level of 0.59 $\mathrm{ng} / \mathrm{mL}$ for the diagnosis of VUR. Thus, it was suggested that a high procalcitonin level may not only be used for all grades of VUR in children with febrile UTI, however, a low level of the biomarker may also be used to preclude unnecessary MCUG in some low-risk patients (18).

Finally, in a more recent report, Barati et al. showed that procalcitonin was an early predictor of renal parenchymal injury in children with UTI (42). A prospective study was carried out in 95 patients who were admitted in the hospital with the first febrile UTI. Serum procalcitonin of all patients was measured while sensitivity, specificity, positive, and negative predictive value of this biomarker were analyzed compared to DMSA scan. Sensitivity, specificity, positive predictive value, and negative predictive value of procalcitonin reported in optimum cut off were $70 \%, 88.1 \%, 88.1 \%$, and $70 \%$, respectively (42).

\section{Other Novel Biomarkers}

Apart from the interleukins and procalcitonin, other novel biomarkers have also been reported as diagnostic and prognostic tools for UTI in children. In a descriptive, cross-sectional study on children (aged 2 months to 14 years) with UTIs' who were admitted in provincial hospital in Iran, the authors evaluated the utility of urine NGAL in predicting renal parenchymal injury (43). Their findings however suggest that urine NGAL was not sensitive enough for the prediction of renal parenchymal injury, but proved to be a specific biomarker.

In a related study, Yilmaz et al. assessed whether urine NGAL could represent a novel, reliable biomarker of UTI, as well as determined the optimal cut-off level for urine NGAL to predict UTI in children (9). The investigators enrolled 60 patients with a symptomatic UTI and 29 healthy controls. DMSA scan was done in patients with a UTI in order to differentiate pyelonephritis from a lower UTI. The major findings include the significantly higher mean urine NGAL level in the UTI group compared to that in the controls, and its sensitivity and specificity of $97 \%$ and $76 \%$, respectively using a cut-off value of $20 \mathrm{ng} / \mathrm{mL}$ for the diagnosis of UTI.

Another novel biomarker, which has been evaluated, is urine IL-1 $\beta$. In a study of 75 children aged 1-121 months with a diagnosis of first-time febrile UTI, the authors assessed some inflammatory markers including CRP and urine IL-1 $\beta$ (19). Again, the findings of the study indicate that the urine IL-1 $\beta$ level may serve as a useful biomarker for the early detection of acute pyelonephritis in febrile children.

Finally, Chien et al. studied the usefulness of urine 8-hydroxy-2'-deoxyguanosine (8-oxodG) and total antioxidant capacity (TAC) in the prediction of renal parenchymal injury in children with UTI (22). Seventy-three children under the age of 5 years were enlisted, in whom DMSA scan was performed to establish any renal injury. Interestingly, patients with positive DMSA scan had higher levels of urine 8-oxodG and higher urine TAC than patients with normal DMSA scan: a finding which suggests that high level of urine 8-oxodG may be a risk factor of severe renal injury (22).

\section{Conclusions}

Although there is now abundant evidence showing the reliability of these biomarkers for evaluating UTI in children (including their acceptable sensitivity and specificity), there are still inconsistent results in the utility of urine IL-6, serum and urine IL-8 for the diagnosis of acute pyelonephritis. Procalcitonin has not only to been shown to be a dependable biomarker in the diagnosis of acute pyelonephritis but also a predictor of renal parenchymal injury and VUR. Obviously, this biomarker can be used in settings where radiological tests like DMSA and MCUG may not be readily available, as well as when there is the need to avoid radiation exposure. Nevertheless, given the wide ranges in sensitivity and specificity values reported for procalcitonin and IL- 6 in the differentiation of lower UTI from pyelonephritis, the diagnostic and prognostic utility of these biomarkers require validation by further research. Thus, the use of imaging studies has not been completely 
eliminated, given their usefulness in detecting structural abnormalities of the urinary tract, which potentially predispose to UTI.

\section{Acknowledgments}

The author acknowledges the invaluable information obtained from the article- Nanda M, Juthani-Mehta M. novel biomarkers for the diagnosis of urinary tract infection a systematic review.

\section{Footnote}

Disclosures: The author reports no conflict of interest.

\section{References}

1. Srivastava RN, Bagga A. In: Pediatric Nephrology. Srivastava RN, Bagga A, editors. New Delhi: Jaypee; 2005. pp. 235-64.Urinary tract infection.

2. Rees L. In: Paediatric Nephrology-Oxford Specialist Handbooks in Paediatrics. Rees L, Brogan PA, Bockenhauer D, Webb NJA, editors. Oxford Oxford University Press; 2012. Urinary tract infection.

3. Shaikh N, Morone NE, Bost JE, Farrell MH. Prevalence of urinary tract infection in childhood: a meta-analysis. Pediatr Infect Dis J. 2008;27(4):302-8. doi: 10.1097/INF.0b013e31815e4122. [PubMed: 18316994].

4. Chang SL, Shortliffe LD. Pediatric urinary tract infections. Pediatr Clin North Am. 2006;53(3):379-400. doi: 10.1016/j.pcl.2006.02.011. [PubMed: 16716786] vi.

5. Larcombe J. Urinary tract infection in children. BMJ Clin Evid. 2010;2010 [PubMed: 21733199].

6. Leroy S, Gervaix A. [Procalcitonin, a useful biomarker in pediatric urinary tract infection]. Arch Pediatr. 2013;20(1):54-62. doi: 10.1016/j.arcped.2012.10.025. [PubMed: 23199564].

7. Leroy S, Gervaix A. Procalcitonin: a key marker in children with urinary tract infection. Adv Urol. 2011;2011:397618. doi 10.1155/2011/397618. [PubMed: 21274426].

8. Pecile P, Romanello C. Procalcitonin and pyelonephritis in children. Curr Opin Infect Dis. 2007;20(1):83-7. doi: 10.1097/QCO.0b013e328013cb2a. [PubMed: 17197887].

9. Yilmaz A, Sevketoglu E, Gedikbasi A, Karyagar S, Kiyak A, Mulazimoglu $\mathrm{M}$, et al. Early prediction of urinary tract infection with urinary neutrophil gelatinase associated lipocalin. Pediatr Nephrol. 2009;24(12):2387-92. doi: 10.1007/s00467-009-1279-6. [PubMed 19649660].

10. Benson M, Jodal U, Agace W, Hellstrom M, Marild S, Rosberg S, et al. Interleukin (IL)-6 and IL-8 in children with febrile urinary tract infection and asymptomatic bacteriuria. J Infect Dis. 1996;174(5):1080-4. [PubMed: 8896512].

11. Kim BH, Yu N, Kim HR, Yun KW, Lim IS, Kim TH, et al. Evaluation of the optimal neutrophil gelatinase-associated lipocalin value as a screening biomarker for urinary tract infections in children. Ann Lab Med 2014;34(5):354-9. doi: 10.3343/alm.2014.34.5.354. [PubMed: 25187887].

12. Daure E, Belanger MC, Beauchamp G, Lapointe C. Elevation of neutrophil gelatinase-associated lipocalin (NGAL) in non-azotemic dogs with urinary tract infection. Res Vet Sci. 2013;95(3):1181-5. doi 10.1016/j.rvsc.2013.09.002. [PubMed: 24074691].

13. Yim HE, Yim H, Bae ES, Woo SU, Yoo KH. Predictive value of urinary and serum biomarkers in young children with febrile urinary tract infections. Pediatr Nephrol. 2014;29(11):2181-9. doi: 10.1007/s00467-0142845-0. [PubMed: 24924751].
14. Leroy S, Adamsbaum C, Marc E, Moulin F, Raymond J, Gendrel D, et al. Procalcitonin as a predictor of vesicoureteral reflux in children with a first febrile urinary tract infection. Pediatrics. 2005;115(6):e706-9. doi: 10.1542/peds.2004-1631. [PubMed: 15867014].

15. Leroy S, Romanello C, Galetto-Lacour A, Bouissou F, Fernandez-Lopez A, Smolkin V, et al. Procalcitonin is a predictor for high-grade vesicoureteral reflux in children: meta-analysis of individual patient data. J Pediatr. 2011;159(4):644-51 e4. doi: 10.1016/j.jpeds.2011.03.008. [PubMed: 21511275].

16. Sun HL, Wu KH, Chen SM, Chao YH, Ku MS, Hung TW, et al. Role of procalcitonin in predicting dilating vesicoureteral reflux in young children hospitalized with a first febrile urinary tract infection. Pediatr Infect Dis J. 2013;32(9):e348-54. doi: 10.1097/INF.ob013e3182905d83. [PubMed: 23584577].

17. Rahimzadeh N, Otukesh H, Hoseini R, Shadani S, Hooman N. Serum procalcitonin level for prediction of high-grade vesicoureteral reflux in urinary tract infection. Iran J Kidney Dis. 2014;8(2):105-8. [PubMed: 24685732].

18. Mortazavi F, Ghojazadeh M. Usefulness of serum procalcitonin level for prediction of vesicoureteral reflux in pediatric urinary tract infection. Iran J Kidney Dis. 2014;8(1):37-41. [PubMed: 24413719].

19. Sheu JN, Chen MC, Cheng SL, Lee IC, Chen SM, Tsay GJ. Urine interleukin-1beta in children with acute pyelonephritis and renal scarring. Nephrology (Carlton). 2007;12(5):487-93. doi: 10.1111/j.14401797.2007.00819.x. [PubMed: 17803473].

20. Leroy S, Fernandez-Lopez A, Nikfar R, Romanello C, Bouissou F, Gervaix A, et al. Association of procalcitonin with acute pyelonephritis and renal scars in pediatric UTI. Pediatrics. 2013;131(5):870-9. doi: 10.1542/peds.2012-2408. [PubMed: 23629615].

21. Sheu JN, Chen MC, Chen SM, Chen SL, Chiou SY, Lue KH. Relationship between serum and urine interleukin-6 elevations and renal scarring in children with acute pyelonephritis. Scand $J$ Urol Nephrol. 2009;43(2):133-7. doi: 10.1080/00365590802478742. [PubMed: 18972261].

22. Chien JW, Wang LY, Cheng YS, Tsai YG, Liu CS. Urinary 8-hydroxy2'-deoxyguanosine (8-oxodG) level can predict acute renal damage in young children with urinary tract infection. Biomarkers. 2014;19(4):326-31. doi: 10.3109/1354750X.2014.910552. [PubMed: 24749909].

23. Benson M, Jodal U, Andreasson A, Karlsson A, Rydberg J, Svanborg C. Interleukin 6 response to urinary tract infection in childhood. Pediatr Infect Dis J. 1994;13(7):612-6. [PubMed: 7970949].

24. Sheu JN, Chen MC, Lue KH, Cheng SL, Lee IC, Chen SM, et al. Serum and urine levels of interleukin-6 and interleukin- 8 in children with acute pyelonephritis. Cytokine. 2006;36(5-6):276-82. doi: 10.1016/j.cyto.2007.02.006. [PubMed: 17374489].

25. Nanda N, Juthani-Mehta M. Novel biomarkers for the diagnosis of urinary tract infection-a systematic review. Biomark Insights. 2009;4:11121. [PubMed: 19707519].

26. National Institute for Health and Clinical Excellence . Urinary Tract Infection in Children. NICE Clinical Guideline 54. London: NICE;2007.

27. Robinson JL, Finlay JC, Lang ME, Bortolussi R. Urinary tract infections in infants and children: diagnosis and management. Paediatr Child Health. 2014;19(6):315-9.

28. Mischak H, Delles C, Vlahou A, Vanholder R. Proteomic biomarkers in kidney disease: issues in development and implementation. Nat Rev Nephrol. 2015;11(4):221-32. doi: 10.1038/nrneph.2014.247. [PubMed: 25643662].

29. Cruz DN, Goh CY, Haase-Fielitz A, Ronco C, Haase M. Early biomarkers of renal injury. Congest Heart Fail. 2010;16 Suppl 1:S25-31. doi: 10.1111/j.1751-7133.2010.00163.x. [PubMed: 20653708].

30. Agace W, Hedges S, Andersson U, Andersson J, Ceska M, Svanborg C. Selective cytokine production by epithelial cells following exposure to Escherichia coli. Infect Immun. 1993;61(2):602-9. [PubMed: 8423089]. 
31. Hedges S, Agace W, Svensson M, Sjogren AC, Ceska M, Svanborg C. Uroepithelial cells are part of a mucosal cytokine network. Infect Immun. 1994;62(6):2315-21. [PubMed: 8188354].

32. Wullt B, Bergsten G, Connell H, Rollano P, Gebratsedik N, Hang L, et al. P-fimbriae trigger mucosal responses to Escherichia coli in the human urinary tract. Cell Microbiol. 2001;3(4):255-64. [PubMed: 11298649].

33. Svanborg C, Agace W, Hedges S, Lindstedt R, Svensson ML. Bacterial adherence and mucosal cytokine production. Ann N Y Acad Sci. 1994;730:162-81. [PubMed: 7915893].

34. Agace WW, Hedges SR, Ceska M, Svanborg C. Interleukin-8 and the neutrophil response to mucosal gram-negative infection.J Clin Invest. 1993;92(2):780-5. doi: 10.1172/JCI116650. [PubMed: 8349817].

35. Jantausch BA, O’Donnell R, Wiedermann BL. Urinary interleukin-6 and interleukin-8 in children with urinary tract infection. Pediatr Nephrol. 2000;15(3-4):236-40. [PubMed: 11149117].

36. Gurgoze MK, Akarsu S, Yilmaz E, Godekmerdan A, Akca Z, Ciftci I, et al. Proinflammatory cytokines and procalcitonin in children with acute pyelonephritis. Pediatr Nephrol. 2005;20(10):1445-8. doi: 10.1007/s00467-005-1941-6. [PubMed: 16079986].

37. Assicot M, Gendrel D, Carsin H, Raymond J, Guilbaud J, Bohuon C. High serum procalcitonin concentrations in patients with sepsis and infection. Lancet. 1993;341(8844):515-8. [PubMed: 8094770].
38. Benador N, Siegrist CA, Gendrel D, Greder C, Benador D, Assicot $\mathrm{M}$, et al. Procalcitonin is a marker of severity of renal lesions in pyelonephritis. Pediatrics. 1998;102(6):1422-5. [PubMed: 9832579].

39. Smolkin V, Koren A, Raz R, Colodner R, Sakran W, Halevy R. Procalcitonin as a marker of acute pyelonephritis in infants and children. Pediatr Nephrol. 2002;17(6):409-12. doi: 10.1007/s00467-001-0790-1. [PubMed: 12107804].

40. Pecile P, Miorin E, Romanello C, Falleti E, Valent F, Giacomuzzi F, et al. Procalcitonin: a marker of severity of acute pyelonephritis among children. Pediatrics. 2004;114(2):e249-54. [PubMed:15286264].

41. Guven AG, Kazdal HZ, Koyun M, Aydn F, Gungor F, Akman S, et al. Accurate diagnosis of acute pyelonephritis: How helpful is procalcitonin? Nucl Med Commun. 2006;27(9):715-21. doi: 10.1097/01.mnm.0000230072.61941.71. [PubMed: 16894326].

42. Barati L, Safaeian B, Mehrjerdian M, Vakili MA. Early prediction of renal parenchymal injury with serum procalcitonin. J Renal Inj Prev. 2016;5(3):108-11. doi: 10.15171/jrip.2016.23. [PubMed: 27689104].

43. Ghasemi K, Esteghamati M, Borzoo S, Parvaneh E, Borzoo S. Predictive Accuracy of Urinary neutrophil gelatinase associated lipocalin (NGAL) for renal parenchymal involvement in Children with Acute Pyelonephritis. Electron Physician. 2016;8(2):1911-7. doi: 10.19082/1911. [PubMed: 27053998] 